\title{
Modelo de Evaluación Integral Sostenible para los Sistemas de Bombeo Fotovoltaicos en Comunidades Rurales: El Limón y El Lagartillo
}

Luis Lorenzo Fuentes Peralta ${ }^{1}$

\section{RESUMEN}

Este artículo es resultado de una investigación realizada, la cual se enfoca inicialmente en los aspectos relacionados con el quehacer de la administración pública la cual entrelaza los niveles de organización, gestión con un enfoque participativo comunitario para la conservación y manejo sostenible del recurso agua administrados en las comunidades rurales por los comités de agua potable y saneamiento (CAPS). El objetivo de investigación fue elaborar un modelo de evaluación integral sostenible para sistemas de bombeo fotovoltaico (SBFv), instalados en las comunidades rurales del Limón del municipio de Estelí y El Lagartillo del municipio de Achuapa del departamento de León. En cada comunidad se construyó una línea de tiempo del proceso de organización el cual inicia desde la conformación de los comités de agua hasta la constitución legal como un CAPS que forma coincidente en ambas comunidades fue en el año 2012. Se realizaron estudios de parámetros eléctricos e hidráulicos de los SBFv de cada comunidad, el modelo propone una matriz check list de evaluación de las condiciones de sistemas agua potable, asimismo se desarrolló una plantilla de cálculo excel para evaluar o dimensionar un nuevo sistema de bombeo para comunidades asiladas de las conexiones de la red eléctrica. La investigación tiene un enfoque cualitativo y cuantitativo (Mixto). Se considera cualitativa por que se basó en la aplicación del instrumento de recolección de datos como lo es el grupo focal con el fin de identificar el nivel de organización y gestión de los CAPS, y es eminentemente cuantitativa, dado que se utilizaron instrumentos de recolección de datos de variables de conteo y medición.

Palabras claves: Administración pública, organización, CAPS, sostenibilidad, bombeo de agua fotovoltaico.

Recibido: 12 de diceimbre de 2016

Aceptado: 16 de febrero de 2017

1 Egresado de la maestria en Gerencia y Administración Publica. UNAN-Managua-FAREM-Estelí. Correo electronico: 1lfuentesp072@yahoo.com.mx 


\title{
Sustainable Integral Evaluation Model for Photovoltaic Pumping Systems in Rural Communities: El Limón and El Lagartillo
}

\begin{abstract}
This article reveals the results of a research focused on the aspects related to the work of the public administration which interweaves the levels of organization, management with a participatory community approach for the conservation and sustainable management of water resources managed in rural communities by drinking water and sanitation committees (CAPS). The research objective was to develop a sustainable integral evaluation model for photovoltaic pumping systems (SBFv), installed in the rural communities of Limón in the municipality of Estelí and El Lagartillo in the municipality of Achuapa of the departament of León. In each community a time line of the organization process was built, starting from the formation of water committees until the legal constitution as a CAPS that forms a coincidence in both communities was in the year 2012 . Electrical and hydraulic parameters of the SBFv of each community were carried out. The model proposes a check list matrix for the evaluation of the conditions of potable water systems, as well as an excel calculation template to evaluate or size a new pumping system for Communities of power grid connections. The research has a mixed methods approach. It is considered qualitative because it was based on the application of the data collection instrument as it is the focal group in order to identify the level of organization and management of the CAPS, and it is eminently quantitative, since instruments of data collection were used data of counting and measuring variables.
\end{abstract}

Keywords: Public administration, organization, CAPS, sustainability, photovoltaic water pumping. 


\section{INTRODUCCIÓN}

La organización y la gestión comunitaria son herramientas de los procesos administrativos presentes en comunidades rurales que manifiestan la capacidad de participación de las personas como una forma de sumar esfuerzos en la consecución de un objetivo común en correspondencia a las necesidades específicas de los comunitarios, por lo cual la participación es un instrumento que confiere el poder a la comunidad para realizar la toma de decisiones basadas en un diagnóstico de necesidades concretas.

Al respecto (Chiavenato, 2007) plantea que "Una organización es un sistema de actividades conscientemente coordinadas de dos o más personas. La cooperación entre estas personas es esencial para la existencia de la organización". Es por lo tanto que la existencia de la organización se da cuando, las personas son capaces de comunicarse, están dispuestas a contribuir en una acción conjunta para alcanzar un propósito o un objetivo común.

Es asimismo, que una comunidad inmersa en el proceso de organización participativa puede llevar a cabo con éxito la gestión comunitaria del recurso agua y maximizar su sostenibilidad en el tiempo, este proceso de gestión participativa se fundamenta en lograr la satisfacción de necesidades más básicas, que entrelazan la subsistencia del ser humano y su calidad de vida en sitios asilados de la interconexión de redes eléctricas e hidráulicas de los pueblos o ciudades.

En referencia a lo anterior, Nicaragua posee altos niveles de incidencia solar que de acuerdo a (Handboock of Industrial Drying, 2006) están entre el rango (4.8 - 5.43 $(\mathrm{kWh} / \mathrm{m} 2$ día). Esto permite que se puedan aprovechar dichas condiciones y hacer viable la implementación de alternativas de generación de energía fotovoltaica para sistemas de bombeos que suministren agua para el consumo humano, saneamiento básico y la sostenibilidad alimentaria en aplicaciones agrícolas.
El modelo de evaluación integral que se presenta en esta investigación tienen incidencia en las comunidades de: El Limón del municipio de Estelí y el Lagartillo del municipio de Achuapa del departamento de León. Y además de presentar ámbitos de organización y gestión de los Comités de Agua Potable y Saneamiento (CAPS), hace referencia a la caracterización de los parámetros hidráulicos y eléctricos necesarios para el estudio de este tipo de alternativas de bombeo con energía autónoma renovable y el impacto del sistema de bombeo fotovoltaico en la calidad de vida de los usuarios.

Sabemos que no es posible de ninguna manera, que un sistema de agua potable por si solo pueda brindar un servicio efectivo a una comunidad, para esto debe anteponerse en primer lugar el factor humano, quien se organiza y establece planes de manejo y gestión administrativa en cada territorio de forma participativa expresada en la conformación de los CAPS, quienes como administradores y según lo descrito por (Erskine Canelo, 2016), “deben cumplir los siguientes cinco aspectos que corresponden a todo proceso administrativo: planear, organizar, integrar, dirigir y controlar". Lo antes descrito es totalmente congruente para que cualquier tipo de proyecto funcione de forma correcta y sea sostenible en el tiempo, prestando las mejores condiciones de disponibilidad a través del uso y el manejo racional de las fuentes de abastecimiento de agua. Por lo tanto es necesario que:

- Los sistemas de bombeo fotovoltaico comunitarios, deben ser evaluados para ver si presentan algunas deficiencias de manejo administrativo por parte de los CAPS.

- Sistemas que funcionan, pero con limitaciones, ya que no han destinado fondos necesarios para la gestión del mantenimiento, cuyos pilares principales son la disponibilidad, confiabilidad y costos accesibles los cuales conllevan a la sostenibilidad del sistema.

- Los CAPS, no reciben capacitación sistemática que 
les permita actualizarse sobre el manejo racional de los sistemas de agua potable.

- Existen algunos sistemas a los cuales nunca se le ha realizado una evaluación del recurso hídrico, para ajustar los niveles freáticos del manto acuífero. Esto ha permitido que se extralimiten los niveles de explotación de los pozos y como consecuencia algunos se han secado.

- Algunos CAPS no realizan trabajo de regeneración natural asistida, reforestación y manejo de micro cuencas.

- No existe un sistema de micro medición para establecer tarifas de pago acordes al consumo de las conexiones domiciliares.

- Existen CAPS que no llevan ningún tipo de registro sobre el caudal de agua bombeado.

- Hay sistemas que no cuentan con proceso de cloración para tratar el agua, y evitar así la propagación de infecciones y enfermedades intestinales agudas en las comunidades.

Actualmente uno de los problemas que apremia a las comunidades rurales, es la falta de energía eléctrica lo cual es una de las limitante principales para el acceso a los sistemas de agua potable, por ello el llevar un buen proceso de gestión y manejo adecuado de sistemas que funcionen a base de energía limpia y amigable con el medio ambiente, no sólo contribuyen a mitigar el cambio climático, sino que se convierten en un elemento motriz dinámico de desarrollo, que se refleja en mejores niveles de vida de las personas en cuanto al recurso agua y saneamiento. El uso de este tipo de energía, evidencia una relación proporcional entre el acceso a la energía y su aprovechamiento para disminuir los niveles de pobreza en las zonas rurales

La investigación, tiene la finalidad de elaborar un modelo de evaluación integral sostenible para sistemas de bombeo solar fotovoltaico (BSFv), en comunidades rurales aisladas de la interconexión de la red eléctrica convencional, el estudio difiere de cualquier otro tipo de trabajo realizado, dado que se aborda desde una perspectiva más amplia, basándose en el análisis de la implementación de las políticas públicas gubernamentales que se fundamentan en la planificación estratégica como un instrumento de gestión de manera que en este caso los lineamientos, convergen en el quehacer de la administración pública, tanto a nivel de gobierno nacional como gobierno municipal, con el fin de atender de manera directa y efectiva, las necesidades puntuales en cada territorio y propiciar de esta forma la organización colectiva y participativa que permita alcanzar el bien común de todo los pobladores.

Bajo esta dinámica, el instrumento de evaluación tiene el objeto de servir de referencia para la evaluación de otros sistemas similares que son administrados por los Comités de Agua Potable y Saneamiento (CAPS), según lo estipulado en la Ley No. 722- Ley Especial de Comités de Agua Potable y Saneamiento (Poder Legislativo, 2010); los CAPS son en sí, organizaciones comunitarias que juegan un rol importantísimo en el uso racional del agua y efectúan un trabajo arduo de sostenibilidad día a día, según (Fandiño, 2014) "El agua es vida, pero también puede ser muerte si no se maneja o se cuida bien, y que para cuidarla hay que ORGANIZARSE".

\section{MATERIALES Y MÉTODO}

El estudio se realizó en la comunidad El Limón están entre los $13^{\circ} 03^{\prime} 02.15^{\prime}$ latitud norte y $86^{\circ} 21^{\prime} 44.80^{\prime}$ " longitud oeste, a una cota altitudinal de 840 m.s.n.m y la otra comunidad objeto de estudio fue el Lagartillo del Municipio de Achuapa del departamento de León, ubicado entre las coordenadas $13^{\circ} .05^{\prime} 058^{\prime}$ ' de latitud norte y $-86^{\circ} .33^{\prime} 484^{\prime \prime}$ longitud, y una cota altitudinal de 640 m.s.n.m.

Según su enfoque filosófico el presente estudio, es de tipo cualitativo y cuantitativo (Mixto). Se considera cualitativa por que se basó en la aplicación de instrumentos de recolección de datos tales como: grupos 
focales para poder conocer la percepción del nivel de organización y gestión de los CAPS, así mismo se les aplicó la encuesta a los usuarios que es un instrumento eminentemente cuantitativo, para la recolección de datos de variables de conteo y de medición.

De acuerdo al proceso es del tipo deductivo, secuencial, probatorio analiza la realidad objetiva, en cuanto al enfoque cualitativo el proceso es también inductivo y analiza múltiples realidades subjetivas que se presentan en la investigación, según (Hernández Sampieri, Fernandez , \& Baptista, 2014, pág. 7) plantea que una de las características de este enfoque es que "utiliza la recolección y análisis de los datos para afinar las preguntas de investigación o revelar nuevas interrogantes en el proceso de interpretación".

El método utilizado es el observacional, el cual consiste el control nulo (no manipulación) de las variables independientes o factores (organización); sino que únicamente se efectuaron medición de las variables o parámetros de interés para este estudio.

De acuerdo al tiempo en que se realizó la investigación, se clasifica de tipo transversal por que las variables objeto de estudio se midieron en un solo periodo de tiempo y no en series sucesivas del mismo (longitudinal).

Y según su nivel de profundidad está investigación, es del tipo exploratorio: debido a que tiene por objeto esencial familiarizarnos con un tema desconocido, novedoso o escasamente estudiado; descriptivo: nos permite caracterizar cómo es y cómo se manifiesta un fenómeno y sus componentes, identifica características del universo de investigación, señala formas de conducta y actitudes del universo investigado, establece comportamientos concretos, descubre y comprueba la asociación entre variables de investigación correlacional; el investigador pretende visualizar cómo se relacionan o vinculan diversos parámetros entre sí, o si por el contrario no existe relación entre ellos. Lo principal de estos estudios es saber cómo se puede comportar una variable conociendo el comportamiento de otra variable relacionada (evalúan el grado de relación entre dos variables).

La población se determinó según el conjunto finito de las comunidades rurales del municipio de Estelí y las comunidades de Achuapa del departamento de León, sitios en donde existen los tipos de sistemas de los tipos Mini Acueducto por Bombeo Eléctrico (MABE), pero que tienen en común una característica bien específica y es que trabajan con fuentes de energías renovables, por lo tanto, poseen características comunes, relacionadas con el estudio en cuestión.

Tabla 1. Sistemas Fotovoltaico Municipio de Estelí y Achuapa

\begin{tabular}{lll}
\hline $\mathbf{N}^{\circ}$ & Nombre del municipio & Nombre de la comunidad \\
\hline 1 & \multirow{2}{*}{ Estelí } & El Limón \\
2 & & Sontule \\
\hline 3 & & El Barro \\
4 & Achuapa & El Guanacaste \\
5 & & El Lagartillo
\end{tabular}

Fuente: Unidades Municipales de Agua y Saneamiento (UMAS).

Para la definición de la muestra para este estudio se toma como base las condiciones propias de estas comunidades, se vuelve difícil puntualizar con todas las personas que habitan en estos hogares, por lo tanto siendo que las comunidades estudiadas no son homogéneas en cuanto a cantidad de familias, el criterio utilizado fue considerar como datos válidos para el estudio un valor mayor a la media poblacional del sitio donde el número de familias es menor, en este caso es la comunidad del Lagartillo que cuenta con 33 hogares, por lo que se consideró realizar 28 encuestas para los usuarios en cada comunidad, con este valor se obtienen resultados representativos en el análisis y es posible establecer términos comparativos al aplicar los instrumentos utilizados para el análisis de la investigación en ambas comunidades. 
Según el tipo de muestreo es no probabilístico e intencionado, porque no fue utilizada ninguna técnica estadística (tómbola y tabla de números estadísticos) para la selección de los encuestados, de manera que la aplicación de la encuesta se realizó a las personas que accedieron de forma voluntaria a responderla.

La recolección de la información utilizada para dar cumplimiento a los objetivos de la investigación fueron las siguientes: Guía de Observación, Encuesta semiestructurada y Grupos focales.

La guía de observación: Consiste en el uso sistemático de nuestros sentidos orientados a la captación de la realidad que queremos estudiar. El tipo de observación aplicada en este estudio es del tipo participante para lo cual se efectúa la integración con la comunidad y el grupo de las juntas directivas de los CAPS, se toman registros y se formaliza el desarrollo del estudio encaminado a seleccionar, organizar y relacionar los datos y aspectos relevantes referentes al problema. Los medios que se utilizaron para registrar la información fueron: cuaderno de campo, diario, computadora portátil, cuadros de trabajo, gráficos y mapas.

Otra técnica que se utilizó para el levantamiento de información es la encuesta con preguntas del tipo semi estructurada. Este instrumento consiste en un cuestionario estructurado aplicado a una muestra de individuos, a quienes se les planteo las mismas preguntas para obtener respuestas sobre el problema de estudio, las personas o usuarios consultadas sobre los sistemas de abastecimiento de agua en cada comunidad, respondieron por voluntad propia a las preguntas del investigador o encuestador. La finalidad de este instrumento es que permite al investigador obtener la información sobre un mayor número de personas en un periodo bastante breve y mediante el mismo se logra cuantificar, el análisis e interpretación de los datos obtenidos.

Asimismo en el estudio se realizaron grupos focales, para lo cual se necesitó de un moderador investigador quien se encargó de iniciar el debate participativo a través de preguntas relacionadas al tema del que hacer de los CAPS para el proceso de organización, gestión y el manejo sostenible de los sistemas de agua potable en cada uno de estos sitios donde tiene incidencia esta investigación, con este instrumento se logró abrir espacios para escuchar las distintas opiniones de las personas que componen la junta directiva del CAPS en cada comunidad.

El proceso de la investigación se realizó con la consolidación efectiva de múltiples fuentes de información con fuerte rigor científico vinculada a la temática de la investigación, se retomó diferentes fuentes de bibliografía especializada, artículos científicos, páginas web y estudios realizados por instituciones, organismos no gubernamentales y universidades. Esta fase se realizó en el transcurso de todas las etapas del proceso de la investigación y permitió la estructuración de la idea de investigación, definición desde la perspectiva teórica en la cual se sustenta; además la indagación del conocimiento y adopción de los métodos científicos necesarios para el desarrollo del proceso investigativo.

Una vez definidos y estructurados los instrumentos se llevó a cabo el trabajo de campo de la investigación, teniendo que coordinar con los presidentes de los CAPS en cada comunidad los permisos necesarios para poder hacer el estudio, las personas consultadas accedieron a la solicitud, de manera que el trabajo de campo consistió en la aplicación de encuestas a usuarios de los sistemas de agua potable en las comunidades, con el objeto de poder precisar el proceso de gestión y organización de los CAPS en cada una de ellas.

Así mismo para cada una de las juntas directiva de los CAPS se hizo uso de las herramientas participativas, como lo es el grupo focal, donde se interactuó de manera activa- participativa en todo momento un dialogo franco y abierto. Para poder llevar acabo esta actividad y lograr obtener datos sobre línea de tiempo, organización a lo interno y externo, esto por medio del 
uso del diagrama de VENN. Con esta técnica se logró conocer el nivel de gestión que se ha realizado en cada uno de los CAPS donde se enfoca este estudio.

Para la evaluación paramétrica se desarrolló con la finalidad de caracterizar los parámetros de los recursos hidráulicos y solares del sistema de bombeo de agua con generación de energía fotovoltaica de manera que se pueda conocer la parte operativa de un sistema de bombeo.

Por lo que para esta etapa de campo, se hizo necesario elaborar formatos de toma de datos técnicos y se trabajó de forma sincronizada en tiempos simultáneos para la toma de datos como caudal bombeo, radiación solar, potencia de generación de energía de producción, comportamiento de niveles de la fuente de agua.

En estos sitios se logró evaluar los parámetros hidráulicos y eléctricos de los sistemas de bombeo solar fotovoltaico (SBFv) instalados, así mismo se determinan los índices de irradiancia para el bombeo, las características eléctricas del generador eléctrico y el acoplamiento entre el generador y el conjunto bomba motor, las pérdidas de potencia, caudal, demanda entre otros.

Un aspectos que se cuidó durante el proceso de la investigación es la validación de los instrumento por medio consultas y revisiones de dos expertos en el tema de estudio con un nivel de experiencia amplio de muchos años de trabajo en sistemas de agua potable primer experto que trabajo en acueductos rurales para el COSUDE y la segunda persona trabaja aun en la coordinación de los CAPS desde la unidad de las UMAS de la municipalidad de Estelí, el primero hizo sus aportes a la encuesta instrumento aplicado a los usuarios y la otra persona experta realizo sus consideraciones para la guía de grupo focal.

La encuesta semiestructurada fue sometida a la prueba de Alfa de Cronbrach para validar su fiabilidad, en el siguiente cuadro se describe el resultado obtenido de un $75.3 \%$ de fiabilidad, valor que está dentro del rango estipulado para un instrumento diseñado para grado de maestría.

Cuadro 2. Prueba de fiabilidad de la encuesta

\begin{tabular}{ll}
\hline \multicolumn{2}{c}{ Estadísticos de fiabilidad } \\
\hline Alfa de Crombach & $\mathrm{N}^{\circ}$ de elementos \\
.753 & 9 \\
\hline
\end{tabular}

Fuente: Elaboración Propia- SPSS

\section{RESULTADOS Y DISCUSIÓN}

Se detallan los principales resultados obtenido de la aplicación de técnicas de recolección de datos (encuesta semi estructurada, guía de observación, grupo focal y toma de parámetros eléctricos e hidráulicos de los sistemas)

A continuación se muestran algunos de los resultados obtenidos por objetivos del estudio realizado en las dos comunidades.

\section{Descripción de la percepción del proceso organizativo de los CAPS y su relación con las entidades vinculadas con el manejo del agua.}

Según las encuestas realizadas y tomando en consideración los criterios de opinión positiva de los usuarios de las comunidad del El Limón y El Lagartillo en referencia a las preguntas relacionadas con la organización, planeación y gestión efectiva de cada uno los CAPS, en el gráfico se ilustra que el nivel de organización para la comunidad del El Limón alcanza un valor del $88.2 \%$ y para la comunidad El Lagartillo el valor obtenido es del $83.9 \%$. En cuanto al nivel de planeación en cada uno de los CAPS el $86.8 \%$ en los encuestados en la comunidad del Limón opinan que se trabaja bajo un plan de gestión definido y así mismo en la comunidad del Lagartillo este valor esta por el orden del $83.5 \%$, en respuesta a la pregunta si su CAPS trabaja efectivamente por el bien común de la comunidad los valores de opinión fueron de 88.9 \% para el Limón y 
del $84.1 \%$ respectivamente, lo que efecto evidencia que los comunitarios tiene un alto nivel de percepción del quehacer de su CAPS en beneficio de sus comunidades y por lo tanto son totalmente consciente de que este factor es determinante, para la búsqueda de soluciones a problemas relacionados a la sostenibilidad del agua tanto en el entorno interno y como gestión a nivel externo con otras entidades relacionadas.

\section{Gráfico $\mathbf{N}^{\circ} 1$. Proceso de organización y gestión de los CAPS en las comunidades El Limón y El Lagartillo}

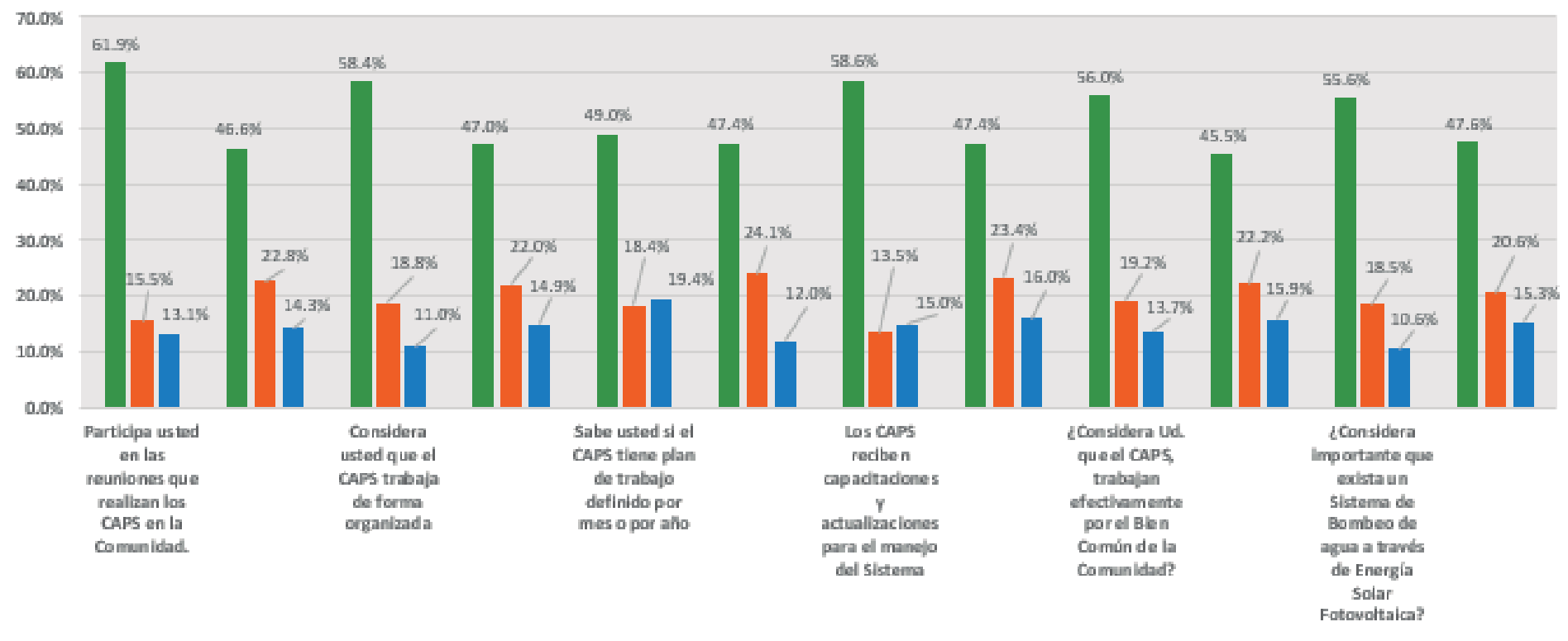

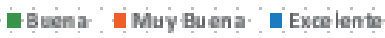

Fuente: Investigación de campo comunidades El Limón y El Lagartillo

Elaborado por: Luis Lorenzo Fuentes Peralta (2016)

Determinación de los parámetros eléctricos e hidráulicos de funcionamiento de los sistemas de bombeo fotovoltaico en los CAPS de comunidades rurales
En los gráficos 2, 3, 4 y 5 se representan los valores de levantamiento de los datos de campo de los parámetros eléctricos e hidráulicos de funcionamiento para los sistemas de bombeo de las comunidades del El Limón y El Lagartillo. El siguiente gráfico ilustra la correlación entre los datos de irradiación y tiempo para la comunidad del Lagartillo.

\section{Gráfico $\mathbf{N}^{\circ}$ 2. Valores de irradiación con Solarímetro PCE-SPM1}

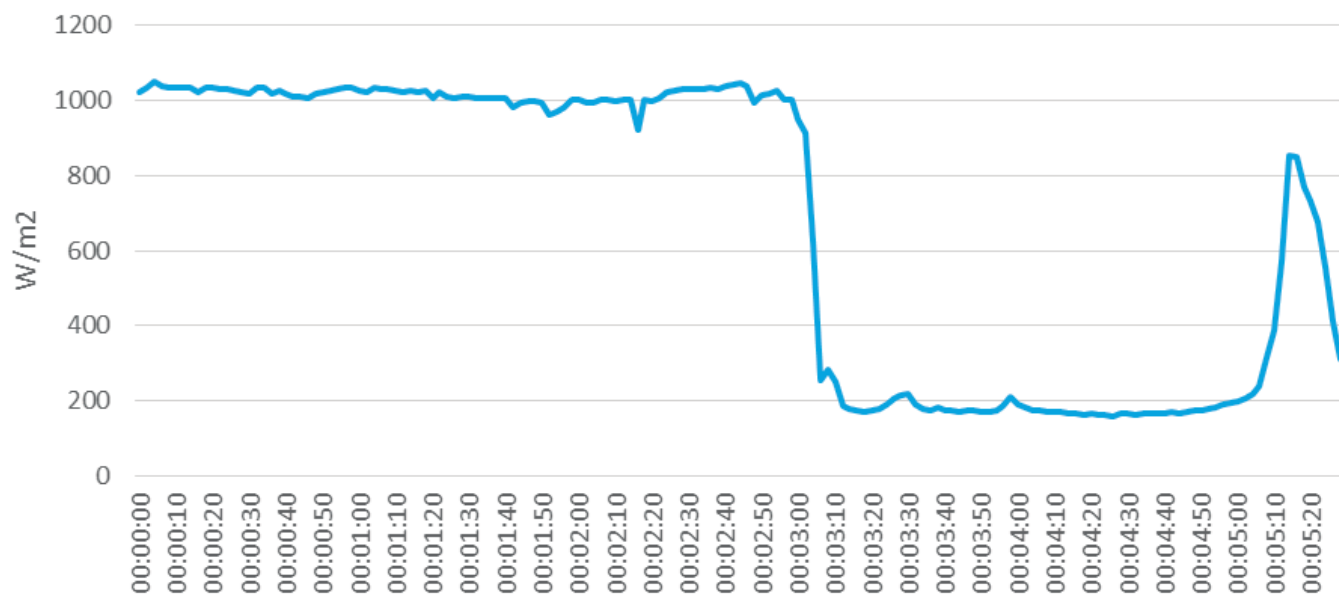

Fuente: Valores de irradiación con Solarímetro PCE-SPM1 
Como se observa en la curva con coordenadas de tiempo e irradiación existen momentos donde los datos se comportan de manera casi constante siendo un poco mayor inclusive al valor estándar de un sol $(1000 \mathrm{~W} / \mathrm{m} 2$ y $25^{\circ} \mathrm{C}$ ), en estos intervalos es cuando la bomba recibe del sistema de generación la potencia pico, pero de igual manera en un periodo de tiempo también se dan caídas rápidas de la irradiancia que alcanza valores de $200 \mathrm{~W} / \mathrm{m} 2$ y en este instante la bomba no funciona, este fenómeno se presenta inclusive con valores menores a $380 \mathrm{~W} / \mathrm{m} 2$. En el trabajo de levantamiento de datos se logró determinar que aun con estas variaciones la bomba genera un caudal promedio de $2.18 \mathrm{~m} 3 / \mathrm{hr}$ bajo su carga dinámica hasta la pila de abastecimiento, este valor fue tomado desde las 10.30Am hasta $11.30 \mathrm{Am}$.

Los datos correspondientes para la comunidad del Limón el siguiente gráfico se muestra los datos sobre la irradiación incidente vrs la potencia para la comunidad del Limón

\section{Gráfico $\mathbf{N}^{\circ}$ 3. Variación de la irradiación vrs potencia comunidad El Limón}

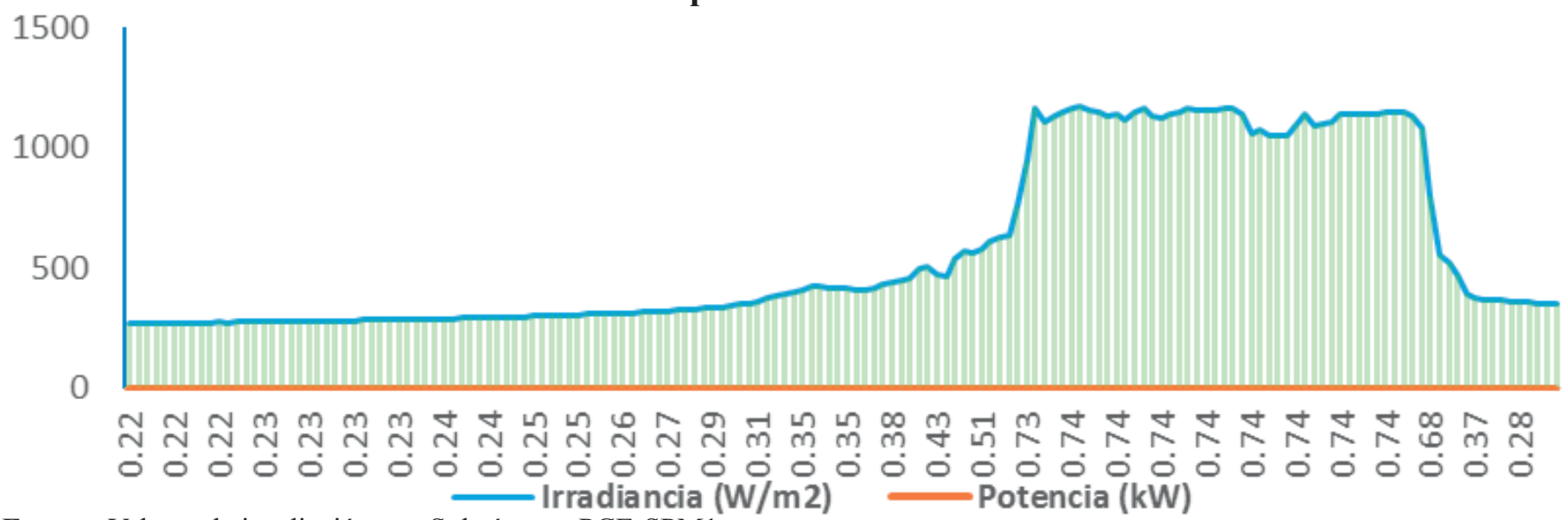

Fuente: Valores de irradiación con Solarímetro PCE-SPM1

La siguiente figura ilustra la correlación que existe entre el caudal de bombeo y la irradiancia incidente

\section{Gráfico $N^{\circ}$ 4. Relación de caudal Vrs Irradiancia comunidad El Limón Estelí}

\subsection{0}

1.20

\section{Relación Caudal vs Irradiancia}

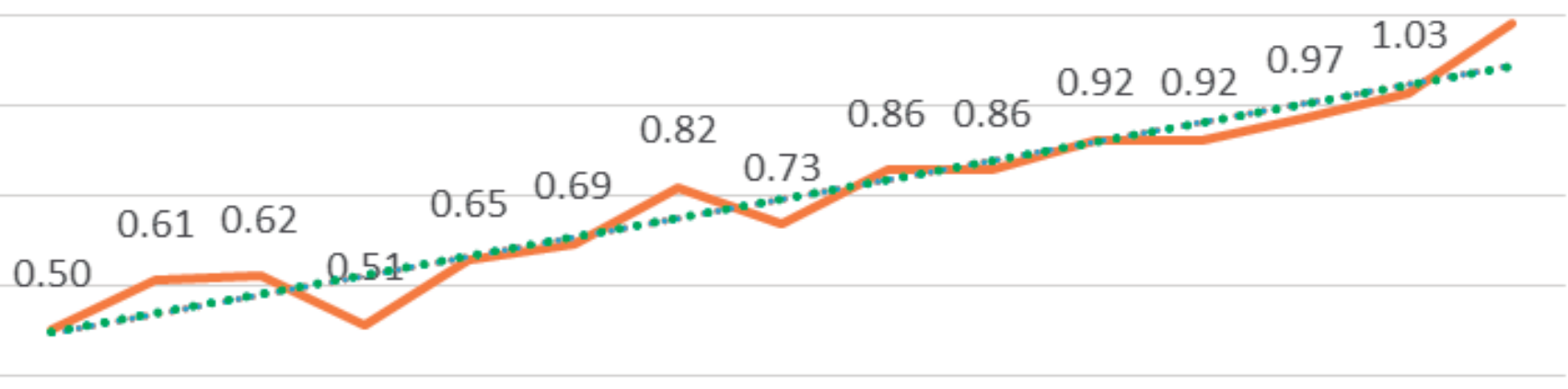

0.20

0.00

\section{$\begin{array}{lllllllllllllll}588 & 618 & 620 & 626 & 631 & 656 & 672 & 684 & 721 & 739 & 751 & 759 & 778 & 795 & 1000\end{array}$ Irradiancia $\left(\mathbf{W} / \mathbf{m}^{2}\right)$}

Fuente: Valores de irradiación con Solarímetro PCE-SPM1 


\section{Correlación de variables}

En el cuadro siguiente se muestra el resultado del grado correlacional de variables entre el caudal e irradiancia, este valor debe estar el rango de $(-1-0+1)$.

Se observa que el grado de asociación $\mathrm{R}$ es de $96.3 \%$ (Pearson), el valor de la determinación es de 92.7\%. Dichos valores resultaron ser muy cercanos a la unidad lo cual indica que si existe un alto valor de correlación entre ambos parámetros y que es de forma proporcional.
Cuadro $\mathrm{N}^{\circ}$ 3. Correlación de variables - Pearson

\begin{tabular}{lllll}
\hline Modelo & $\mathbf{R}$ & $\begin{array}{l}\mathbf{R} \\
\text { Cuadrado }\end{array}$ & $\begin{array}{l}\text { R Cuadrado } \\
\text { corregida }\end{array}$ & $\begin{array}{l}\text { Error } \\
\text { tipo de la } \\
\text { estimación }\end{array}$ \\
\hline 1 & $.963^{\text {a }}$ & .927 & .854 & .24637 \\
\hline
\end{tabular}

Fuente: Elaboración Propia- SPSS

Nota: Para valores promedios de Irradiación $=1000$ $\mathrm{W} / \mathrm{m} 2$ el caudal medio $=1.2 \mathrm{~m} 3 / \mathrm{h}$

Por otro lado la siguiente ilustración representa el comportamiento del sistema de bombeo en relación a las coordenadas tiempo e irradiancia.

\section{Gráfico $N^{\circ} 5$. Variación de la irradiación vrs tiempo comunidad El limón}

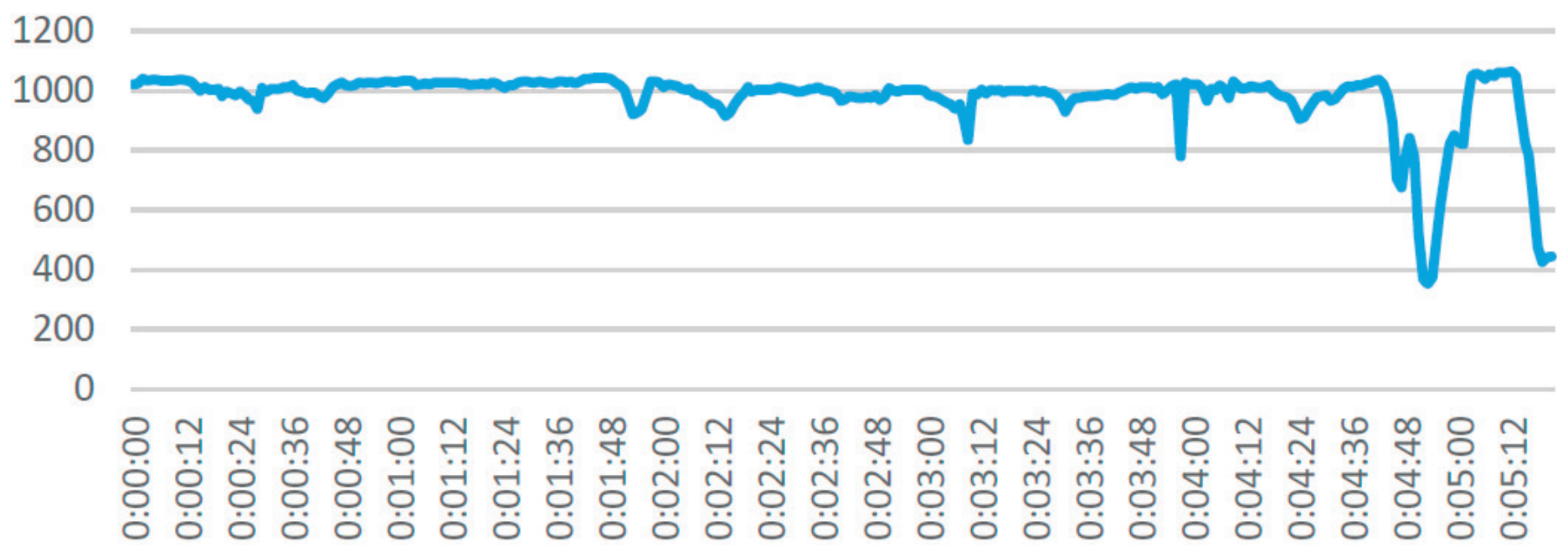

Fuente: Valores de irradiación con Solarímetro PCE-SPM1

Determinación del impacto del sistema de bombeo fotovoltaico en la calidad de vida de los usuarios desde el punto de vista organizativo y técnico

Para determinar el impacto en la calidad de vida de los usuarios que cuentan con un sistema de bombeo fotovoltaico se abordan aspectos tales como la organización y gestión del CAPS, el medio ambiente e higiene y salud.

El siguiente cuadro, refleja según el género encuestado que la cantidad mujeres consultadas en la comunidad del El Limón fue del 78.6\% y que en la comunidad del Lagartillo el resultado fue de $64.3 \%$, el resto corresponden a la población de varones respectivamente para cada una.

Desde el punto de vista del manejo racional del agua y retomando el uso cotidiano que las mujeres le dan a este recurso para realizar sus diferentes quehaceres en el hogar, es relevante conocer desde esta perspectiva la opinión que tienen las mismas.

De acuerdo (Muñoz, S., 2013 citado en (Zavala, 2016) en Nicaragua los Comités de Agua Potable y Saneamiento (CAPS), se han definido como formas de organización comunitaria de hombres y mujeres electos por la comunidad, que se encargan de la gestión del agua, organizando a la población... 
En la ilustración, se observa que los comunitarios piensan que la composición de la junta directiva en cuanto a equidad de género del total de los 28 encuestados de la comunidad El Limón, opinaron que el nivel de distribución de cargos en la junta directiva en cuanto a género es del $78.6 \%$, mientras que en la comunidad El Lagartillo este valor alcanza un $92.8 \%$ el es totalmente favorable a que existan mujeres que integren la junta directiva del CAPS y este resultado es congruente, dado que su presidenta es la señora Juanita Pérez, quien ha efectuado un trabajo encomiable en la gestión del agua para su comunidad.

\section{Gráfico $N^{\circ}$ 6. Composición por género en la junta directiva del CAPS}

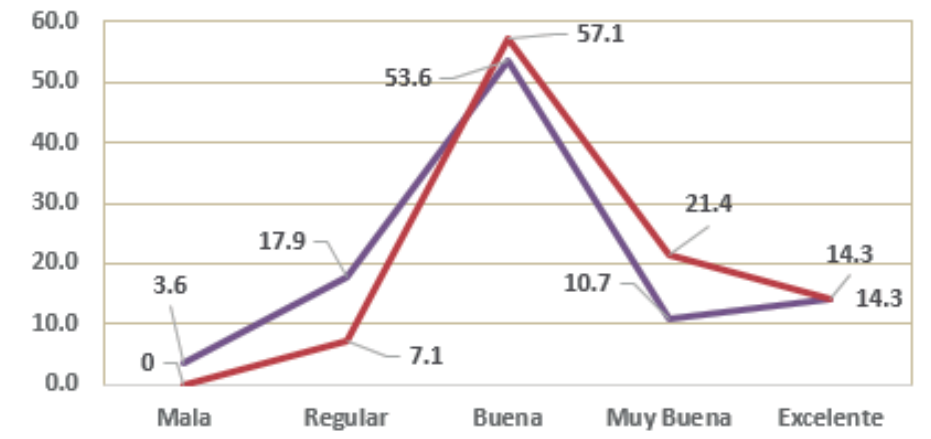

Fuente: Investigación de campo comunidades El Limón y el Lagartllo

La fase de capacitación o entrenamiento es el proceso de desarrollo de capacidades en los recursos humanos para habilitarlos, con el fin de que sean más productivos y contribuyan mejor a la consecución de los objetivos organizaciones. (Chiavenato, 2002)

\section{Cuadro 4. Capacitaciones por género en los CAPS}

\begin{tabular}{|c|c|c|c|c|c|c|}
\hline \multicolumn{2}{|c|}{$\begin{array}{c}\text { Comunidad } \\
\text { donde vive el } \\
\text { encuestado }\end{array}$} & Sexo & Frecuencia & Porcentaje & $\begin{array}{c}\text { Porcentaje } \\
\text { valido }\end{array}$ & $\begin{array}{l}\text { Porcentaje } \\
\text { acumulado }\end{array}$ \\
\hline \multirow{3}{*}{ El Limón } & \multirow{3}{*}{ Válido } & Hombre & 6 & 21.4 & 21.4 & 21.4 \\
\hline & & Mujer & 22 & 78.6 & 78.6 & 100.0 \\
\hline & & Total & 28 & 100.0 & 100 & \\
\hline \multirow{3}{*}{$\begin{array}{l}\text { El } \\
\text { Lagartillo }\end{array}$} & \multirow{3}{*}{ Válido } & Hombre & 10 & 35.7 & 35.7 & 35.7 \\
\hline & & Mujer & 18 & 64.3 & 64.3 & 100.0 \\
\hline & & Total & 28 & 100.0 & 100.0 & \\
\hline
\end{tabular}

Fuente: Elaboración propia
La ilustración, refleja que efectivamente los CAPS reciben capacitaciones y actualizaciones sobre elmanejo y uso racional del agua y este proceso normalmente viene por parte de las alcaldías, a través de las unidades municipales del agua y saneamiento (UMAS). Los CAPS se han formado en aspectos de la Ley 722 Ley Especial de los Comités de Agua y Saneamiento, aprobada desde año 2010, así mismo a nivel territorial tienen incidencia el Ministerio de Salud en cuanto a higiene y salud en las comunidades. Los resultados en grados porcentuales fueron de un $70.4 \%$ para El Limón y para la comunidad del Lagartillo fueron de un $89.3 \%$, en este sentido la última comunidad está más fortalecida en cuanto a formación y es un factor que se evidencia al conversar con las personas que conforman la junta del CAPS.

\section{Gráfico $N^{\circ}$ 7. Los CAPS reciben capacitaciones y actualizaciones para el manejo del sistema}

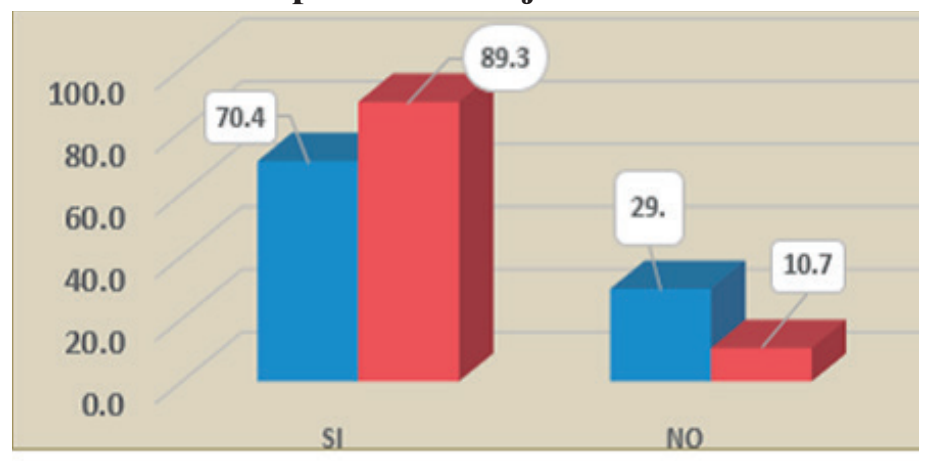

Fuente: Investigación de campo comunidades El Limón y el Lagartllo

El siguiente gráfico refleja el nivel porcentual de valores de opinión favorable que tienen las personas de la comunidad del Limón, las cuales indican que en un $92.8 \%$ su CAPS ha venido realizando un excelente trabajo en cuanto, así mismo para la comunidad del Lagartillo las personas encuestadas valoraron en un $89.4 \%$ que el trabajo realizado por su CAPS es muy bueno. 
Gráfico $N^{\circ} 8$. ¿Cómo considera el trabajo realizado por los CAPS?

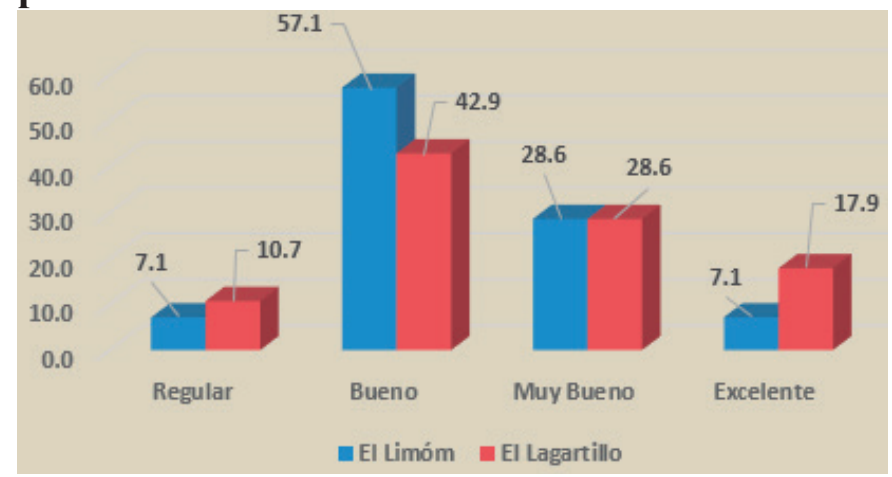

Fuente: Investigación de campo comunidades El Limón y el Lagartllo

Por lo que en base a lo anterior y sin duda alguna en cada comunidad se reconoce que el trabajo desarrollando por cada uno de los CAPS en función del manejo sostenible del agua como un recurso finito es muy bueno.

En la ilustración se refleja el nivel de consideración que tienen los comunitarios, en cuanto a si su CAPS, trabaja efectivamente por el bien común de la comunidad.

Para la comunidad del Limón de los 28 hogares encuestados 26 de ellas respondieron que su CAPS trabaja efectivamente por el bien de la comunidad. Por otro lado, en la comunidad el Lagartillo 27 hogares de un total de 28 encuestados respondieron con propiedad que su comité de agua potable y saneamiento trabaja efectivamente por el bien su comunidad.

Gráfico $N^{\circ}$ 9. ¿Considera usted que los CAPS trabajan efectivamente por el bien común de la comunidad?

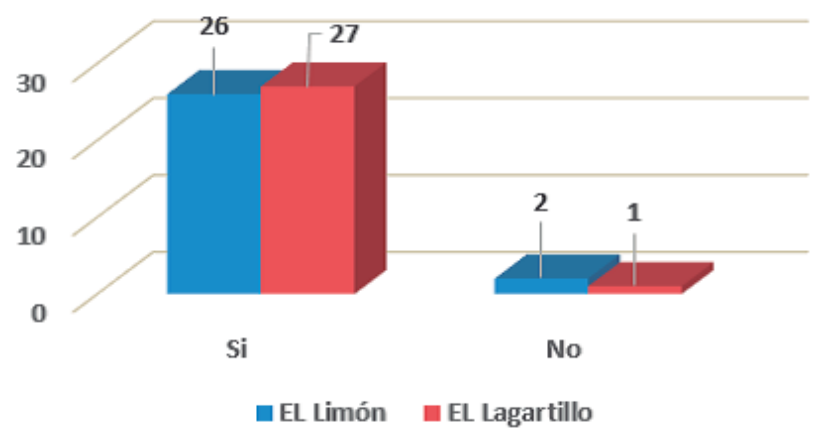

Fuente: Investigación de campo comunidades El Limón y el Lagartllo
Según (Calva , 2009) la satisfacción se entiende como la razón, acción o modo con que se sosiega y responde enteramente a una queja, sentimiento o razón contraria. Nuestra sensación de estar satisfecho, la reducimos, por tanto, a lo que nos es grato, próspero o bien nos conduce a sentirnos complacidos o simplemente contentos.

Las personas de estas comunidades saben que, el comité de agua potable es un valuarte de su comunidad, y ellos están empoderados del trabajo cohesionado que estos efectúan día con día para la conservación y correcta operación de cada uno del sistema.

Al observar la figura pareciera ser que la comunidad del Lagartillo no está satisfecha con el servicio de distribución de agua y en definitiva existe un grado de insatisfacción comparándolo con los resultados de la comunidad del Limón. Por lo que sumando las consideraciones positivas se obtiene que un $82.1 \%$, este dato es alto, tomando en consideración que en épocas de verano tienen que racionar y solo cuentan con un servicio de 35 a 45 minutos diarios de agua por familia, esto debido al poco volumen de agua que genera la fuente debido a que su nivel disminuye a valores muy críticos en dicha temporada. No ocurre lo mismo para la comunidad El Limón donde el nivel de opinión alcanzo un valor del $92.8 \%$, que refleja el alto nivel de satisfacción los usuarios dado que cuentan con un suministro de agua durante las 24 hrs del día.

\section{Gráfico $\mathrm{N}^{\circ} 10$. Grado de santificación con el servicio} de distribución de agua potable

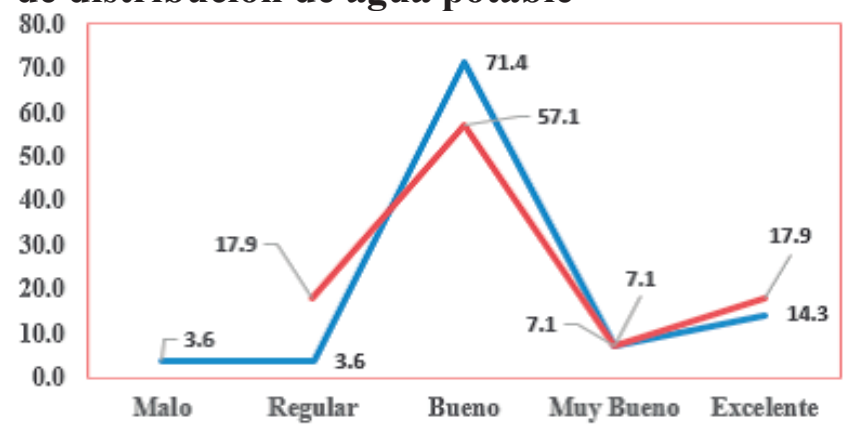

—El Limón _El Lagartillo

Fuente: Investigación de campo comunidades El Limón y el Lagartllo 
Para (Posorky, 1996), "esto no es solo debido al ahorro energético, sino también a que en los sitios más remotos puede resultar económicamente más viable instalar un equipo de estas características que llevar una línea desde la red".

En el siguiente gráfico se observa que el $96.4 \%$ de los 28 hogares encuestados de la comunidad del EL Limón consideran que es importante que exista un sistema de bombeo de agua fotovoltaico, de igual manera el mismo criterio de opinión positiva es el manifestaron los hogares encuestados en la comunidad del El Lagartillo de Achuapa León.

\section{Gráfico $\mathrm{N}^{\circ}$ 10. Considera importante que exista} un sistema de bombeo de agua a través de energía solar fotovoltaica

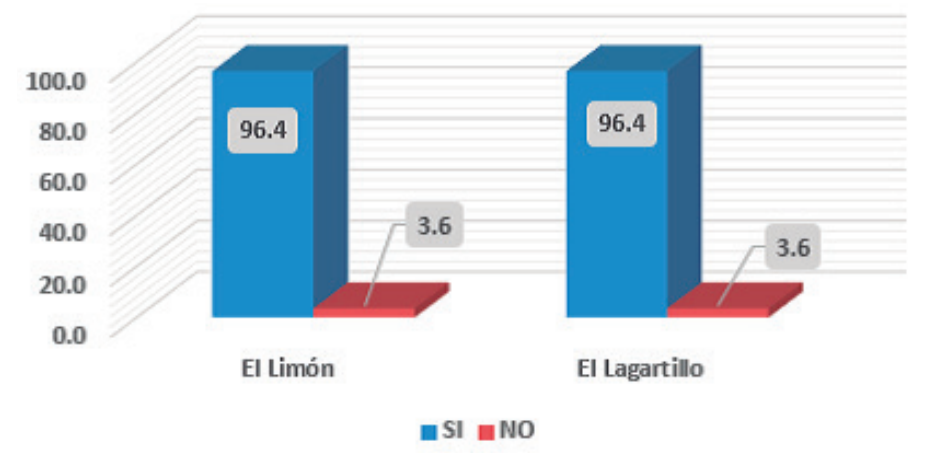

Fuente: Investigación de campo comunidades El Limón y el Lagartllo

Es importante remarcar que hoy en día una de las políticas públicas del gobierno se enfoca en la transformación de la matriz energética y cada sistema por muy pequeño o muy grande que sea aporta en este sentido. Pero sin duda alguna las energías renovables van tomando un lugar preponderante como alternativas sustentables de energía, máxime que pueden ser utilizadas en cual quiere sitio siempre y cuando exista un nivel de irradiación aceptable, por lo menos con valores superior a los $440 \mathrm{de} \mathrm{W} / \mathrm{m} 2$, valor que permite el arranque del equipo de manera que aquellos sitios donde la irradiación es del orden de los $800 \mathrm{~W} / \mathrm{m} 2$ son efectivos el uso de esta tecnologías en dependencia de la cantidad de agua que se requiera bombear.

Proponer un modelo de evaluación integral sostenible, para sistemas de bombeo fotovoltaico de agua en comunidades rurales

Para el modelo de evaluación integral sostenible de los sistemas de bombeo tipo Mini Acueducto de Bombeo Eléctrico (MABE) con generación fotovoltaica implementado en comunidades rurales, contempla un matriz tipo check list que permite conocer de manera rápida el estado funcional de estos miniacuedos manejados por los CAPS, dicha matriz esta categorizada por puntos definidas por estrellas, asimismo se describen las plantillas excel de evaluación técnica utilizando un algoritmo combinado de fórmulas, que permite ingresar datos de parámetros hidráulicos y por medio de fórmulas algorítmicas se obtienen los resultados que sirven para evaluar un sistema existente o bien dimensionar un sistema de bombeo nuevo haciendo uso de la energía fotovoltaica.

Check List de Evaluación de los Sistemas de Bombeo Solar Fotovoltaico administrados por los Comités de Agua Potable y Saneamiento (CAPS)

Cuadro $N^{\circ}$ 3. Propuesta de evaluación de sistemas de agua potable comunitarios

\begin{tabular}{ll}
\hline \multicolumn{1}{c}{ Ejes } & \multicolumn{1}{c}{ Acciones } \\
\hline \multirow{3}{*}{ Género } & $\begin{array}{l}\text { Participación de las mujeres en la junta directiva y } \\
\text { toma de decisiones } \\
\text { Participación de la mujer en las asambleas } \\
\text { generales de pobladores }\end{array}$ \\
\hline
\end{tabular}


Cuentan con libros de actas

Plan definido de trabajo

Organización

y gestión del

CAPS

Tienen un reglamento interno

Cuentan con un libro contable

Articulación con instituciones del estado y

organismos no gubernamentales

El CAPS esta debidamente registrado ante la alcaldía del INAAA

Acceso constante al servicio de agua

Funcionamiento Sistema de cobro con recibos oficiales

Ahorro de agua y tratamiento de las aguas servidas

Mantenimiento del sistema de bombeo

Estado del sistema de bombeo

Estado de la captación

Cerca perimetral de la fuente de agua

Infraestructura Puntos de medición de niveles

y sistema Protección perimetral de los sistemas de

de bombeo generación fotovoltaica

fotovoltaico Estado actual de la pila de almacenamiento

Fugas visibles en el sistema de bombeo y red de distribución

Estado actual de las llaves de chorro

Ubicación de paneles (Dirección sur)

Datos de niveles dinámicos y estático

Sistemas de Sarta con macro medición

medición Sarta con punto para prueba de descarga libre

Conexiones domiciliares con macro medición

Reforestación en las fuentes de agua

El invierno con respecto al año anterior como se

ha comportado por lo de la recarga hidráulica de

Medio ambiente fuente

Sistema de cloración del agua

Control de analisis físico-quimico y

bactecnologicos por parte del ENACAL

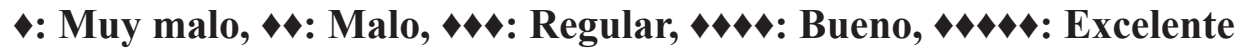

Fuente: Elaboración propia (2016)

Cuadro $\mathrm{N}^{\circ}$ 4. Matriz excel de datos técnicos para calculo hidráulico de bombeo de agua Datos de campo y documentación técnica

\begin{tabular}{|c|c|c|c|}
\hline Denominación & Variable [Unidad] & Valor Formula & Comentario \\
\hline Nivel dinámico & $\mathrm{ND}[\mathrm{m}]$ & 26.2 & Dato obtenido con sonda de Nivel \\
\hline Nivel Estático (NEA) & $\mathrm{NE}[\mathrm{m}]$ & 24.3 & Dato obtenido con sonda de Nivel \\
\hline Altura z_2 & $\mathrm{z} \_2[\mathrm{~m}]$ & 28 & Dato obtenido con GPS \\
\hline Abatimiento & $\mathrm{Ab}[\mathrm{m}]$ & 1.9 & \\
\hline
\end{tabular}




\begin{tabular}{|c|c|c|c|c|}
\hline $\begin{array}{l}\text { Coeficiente de } \\
\text { rugosidad del PVC }\end{array}$ & $\varepsilon[\mathrm{m}]$ & $1.5 \mathrm{E}-06$ & $3.41686 \mathrm{E}-05$ & Fuente: miliarium.com \\
\hline Diámetro interno & $\mathrm{d}$ _interno $[\mathrm{m}]$ & 0.0439 & $f=\frac{1,325}{\left[\ln \left(\frac{\varepsilon}{3,7 * d_{\text {intero }}}+\frac{5,74}{R e^{0,9}}\right)\right]^{2}}$ & \\
\hline $\begin{array}{l}\text { Coeficiente de fricción - } \\
\text { diagrama de Moody }\end{array}$ & $\mathrm{f}$ & $3.15 \mathrm{E}-02$ & $f=0,3164 * R e^{-0,25}$ & Libro de Frank White VI - 6 \\
\hline $\begin{array}{l}\text { Coeficiente de fricción } \\
\text { según Blasius para } \\
\operatorname{Re}<100000\end{array}$ & $\mathrm{f}$ (Blasius) & $3.21 \mathrm{E}-02$ & $d_{\text {nominal }}=1,5 * 0,0254$ & Fuente: miliarium.com \\
\hline Diámetro nominal & d_nominal [m] & 0.0381 & & \\
\hline $\begin{array}{l}\text { Conversión pulgada - } \\
\text { metros }\end{array}$ & una pulgada [m] & 0.0254 & & \\
\hline Coeficiente de pérdidas & $\kappa[\mathrm{m}]$ & 3.77 & & Estimado \\
\hline Longitud del tubo & $1[\mathrm{~m}]$ & 480 & & \\
\hline Eficiencia & $\eta$ & 0.42 & & $\begin{array}{l}\text { De tabla para motor Ac o } \\
\text { CC sumergible con Bomba } \\
\text { Centrífuga }\end{array}$ \\
\hline Densidad de agua & $\rho \_$agua $\left[\mathrm{kg} / \mathrm{m}^{\wedge} 3\right]$ & 1000 & & \\
\hline Gravedad & $\mathrm{g}\left[\mathrm{m} / \mathrm{s}^{\wedge} 2\right]$ & 9.81 & & \\
\hline Viscosidad & $v \_$agua $[\mathrm{m} 2 / \mathrm{s}]$ & $1.50 \mathrm{E}-06$ & & Libro de Frank White VI - 18 \\
\hline
\end{tabular}

Fuente: Elaboración propia (2016)

Cuadro $N^{\circ}$ 5. Matriz excel para calculo hidráulico de bombeo de agua

\begin{tabular}{|c|c|c|c|c|}
\hline \multicolumn{5}{|c|}{ Datos de campo y documentación técnica } \\
\hline Denominación & Variable [Unidad] & Valor & Formula & Comentario \\
\hline Nivel dinámico & $\mathrm{ND}[\mathrm{m}]$ & 26.2 & & Dato obtenido con sonda de Nivel \\
\hline Nivel Estático (NEA) & $\mathrm{NE}[\mathrm{m}]$ & 24.3 & & Dato obtenido con sonda de Nivel \\
\hline Altura z_2 & $\mathrm{z} \_2[\mathrm{~m}]$ & 28 & & Dato obtenido con GPS \\
\hline Abatimiento & $\mathrm{Ab}[\mathrm{m}]$ & 1.9 & & \\
\hline $\begin{array}{l}\text { Coeficiente de } \\
\text { rugosidad del PVC }\end{array}$ & $\varepsilon[\mathrm{m}]$ & $1.5 \mathrm{E}-06$ & $3.41686 \mathrm{E}-05$ & Fuente: miliarium.com \\
\hline Diámetro interno & d_interno $[\mathrm{m}]$ & 0.0439 & $f=\frac{1,325}{\left[\ln \left(\frac{\varepsilon}{3,7 * d_{\text {intero }}}+\frac{5,74}{\left.R e^{0,9}\right)}\right]^{2}\right.}$ & \\
\hline $\begin{array}{l}\text { Coeficiente de fricción - } \\
\text { diagrama de Moody }\end{array}$ & $\mathrm{f}$ & $3.15 \mathrm{E}-02$ & $f=0,3164 * R e^{-0,25}$ & Libro de Frank White VI - 6 \\
\hline $\begin{array}{l}\text { Coeficiente de fricción } \\
\text { según Blasius para } \\
\operatorname{Re}<100000\end{array}$ & $\mathrm{f}$ (Blasius) & $3.21 \mathrm{E}-02$ & $d_{\text {nominal }}=1,5 * 0,0254$ & Fuente: miliarium.com \\
\hline Diámetro nominal & d_nominal $[\mathrm{m}]$ & 0.0381 & & \\
\hline $\begin{array}{l}\text { Conversión pulgada - } \\
\text { metros }\end{array}$ & una pulgada $[\mathrm{m}]$ & 0.0254 & & \\
\hline Coeficiente de pérdidas & $\kappa[\mathrm{m}]$ & 3.77 & & Estimado \\
\hline Longitud del tubo & $1[\mathrm{~m}]$ & 480 & & \\
\hline
\end{tabular}



Eficiencia
$\eta \quad 0.42$
De tabla para motor Ac o
CC sumergible con Bomba
Centrífuga
Densidad de agua
$\rho \_$agua $\left[\mathrm{kg} / \mathrm{m}^{\wedge} 3\right] \quad 1000$
Gravedad
$\mathrm{g}\left[\mathrm{m} / \mathrm{s}^{\wedge} 2\right]$
9.81
Viscosidad
$v \_$agua $[\mathrm{m} 2 / \mathrm{s}]$
$1.50 \mathrm{E}-06$
Libro de Frank White VI - 18

Fuente: Elaboración propia (2016)

\section{CONCLUSIONES}

En las comunidades estudiadas la necesidad del agua es un factor común denominador, que ha permitido en cada una ellas articular esfuerzos y acciones colectivas que se han desarrollado gradualmente en estos sitios rurales. En las comunidades de E1 Limón y E1 Lagartillo a lo largo de una línea de tiempo se han dado eventos muy similares basados en necesidades comunes. Sin duda alguna este proceso ha permitido a cada uno de los comités de agua y saneamiento consolidar los aspectos organizativos y de gestión del agua para sus comunidades.

El estudio permite evidenciar que existe una diferencia sustancial entre las comunidades del El Limón y El Lagartillo, donde la primera de ellas se ha convertido en un sitio semirural condición que facilita al CAPS de esta comunidad, debido a que al tener a disposición mayores números de contactos han podido concretar mayor apoyo financiero y técnico para la sostenibilidad del sistema de agua potable, pero a la vez dicha condición crea un efecto de conformismo entre los comunitarios. Esto no es el caso para la comunidad de El Lagartillo donde las condiciones geográficas son muy distintas, pero un elemento importante de enfatizar es que entre todos los comunitarios existe un factor de sinergia hacia el trabajo colectivo donde las personas tienen un nivel de disposición, participación y empoderamiento hacia la conservación y el manejo racional de su sistema de agua potable.

En cuanto a la tecnología de los sistemas de bombeo fotovoltaico, estos tipo de sistemas ya tiene algún tiempo de implementación, pero cada vez más dicha alternativa es ampliamente utilizada para brindar soluciones a problemas de abastecimiento de agua en sitios aislados, es por lo tanto que la investigación propone a través de una plantilla de cálculos determinar los parámetros hidráulicos y así mismo se detalla la memoria algorítmica para determinar los parámetros eléctricos del dimensionado de un sistema de generación fotovoltaica, estos parámetros servirán de insumo para la evaluación de un sistema en pleno funcionamiento o bien el dimensionado de un sistema nuevo siempre y cuando se conozcan los valores de demanda de agua, según población actual y futura proyectada, datos sobre diferencia de niveles de bombeo, nivel permisible de explotación de la fuente, datos técnicos del recurso solar incidentes en el sitio de estudio.

A través de la aplicación del instrumento de la encuesta semi estructurada, se logró conocer el nivel de percepción que tienen cada uno de los usuarios sobre el trabajo que realizan los CAPS en sus comunidades, ellos valoran que la estructura de esta organización, trabaja efectivamente por el bien común de su comunidad y desde mi perspectiva puedo expresar, que los CAPS son organizaciones baluartes que hacen un trabajo sumamente memorable, siendo agentes de cambio hacia la trasformación y desarrollo de las comunidades rurales.

Como un último aspecto a resaltar es que se logró confirmar la hipótesis planteada en la investigación y por lo tanto se da por aceptada, dado que los resultados obtenidos evidencian que el proceso de organización 
y gestión es un instrumento eficaz que contribuye al correcto funcionamiento de los sistema de agua potable comunitarios, así mismo se concluye que existe correlación de variables (Pearson), dado que el valor obtenido entre las variables caudal e irradiación fue del 0.963 .

\section{RECOMENDACIONES}

\section{Recomendaciones para la comunidad del El Limón}

Se debe establecer un plan de trabajo que estipule la programación de reuniones periódicas con los usuarios para escuchar sus opiniones y que acciones se tienen que tomar para hacer las mejoras de inversión en el sistema.

- Deben actualizar los libros de control que se llevan.

- Realizar a lo inmediato una asamblea con los usuarios para definirles que no pueden seguir usando el agua de la red para otros fines que no sea el consumo y la higiene del hogar, hay consumos promedios mensuales de $542.87 \mathrm{~m} 3$. que significa un consumo por hogar de $11 \mathrm{~m} 3 / \mathrm{mes}$

- Se debe establecer tarifas diferenciadas en función del consumo mensual de cada usuario, dado que existen algunos usuarios que consumen más de $30 \mathrm{~m} 3 / \mathrm{mes}$ que es un consumo excesivo para comunidades rurales.

- Debe programarse el trabajo de extraer el equipo de bombeo y profundizarlo en al menos unos tres metros de tubería y esto debe ser un trabajo coordinado con la comunidad. En los en el periodo 2005 al 2016 el nivel estático ha pasado de 18 $\mathrm{m}$ a $24.2 \mathrm{~m}$ y el NDA ha paso de $20 \mathrm{~m}$ a $26.6 \mathrm{~m}$, esto indica que la reducción del manto o fuente es progresiva y se está agotando y si no se actúa pronto es posible se queme el motor por falta de niveles en la fuente.

- Gestionar ante el ENACAL, apoyo institucional para resolver a aquellos problemas que tengan un nivel de resolución más complejo.

- Gestionar otras fuentes de financiamiento para hacer las mejoras en el sistema de generación fotovoltaica, dado que la inclinación del arreglo no está en la dirección sur que es la correcta donde tendrían mayor nivel de aprovechamiento de la energía solar.

- Gestionar fondos para hacer una conmutación manual que les permita asilar la conexión convencional de la energía directa del generador fotovoltaico

- Gestionar fondos para construir una sarta que lleve punto de descarga libre e instalar un manómetro en la línea de descarga.

- Gestionar participación en procesos de capacitación relacionados a mantenimiento, higiene y salud, manejo de libros contables etc.

\section{Recomendaciones para la comunidad del Lagartillo}

Para este sitio las recomendaciones son meramente técnicas, dado que, en la parte de gestión y organización, trabajo colectivo con los comunitarios ellos están muy bien posicionados, su problema es debido a su ubicación geográfica donde el agua es muy escasa en tiempo de verano. Se les recomienda:

- En el plan de trabajo comunitario que cada hogar tiene realizar para poner en marcha el sistema de bombeo se sugiere observar en invierno el nivel de reboso de la pila para no hacer funcionar la bomba de forma innecesaria.

- Deben gestionar la instalación del sistema de control de niveles para proteger el equipo de bombeo, dado que actualmente el equipo no tiene ninguna protección.

- Al sistema de control y mando se le debe construir una caseta para que no esté a la intemperie porque por la humedad o infiltración de agua podría sufrir daños y esto ya les paso una vez.

- Construir en la sarta de descarga una línea para descarga libre que permita limpiar el pozo en caso de ser necesario.

- Trabajar en un plan de gestión ante ENACAL, para 
que les realice un estudio de pre factibilidad de proyecto para instalar las conexiones domiciliares con tuberías PVC.

- Gestionar de igual manera la instalación del sistema de micro medición de agua para cada usuario.

- Gestionar fondos para cambiar el macro medidor dado que está mal estado

- Hacer limpieza donde está el sistema de generación fotovoltaica para evitar las sobras sobre las células, debido a que estas interfieren el sistema de generación porque están conectadas en serie y la sombra incide sobre algunos módulos fotovoltaicos.

- Hacer gestiones ante INTUR para que más personas conozcan de este lugar en cual las personas son sumamente cálidas y muy amables.

\section{BIBLIOGRAFÍA}

Calva , J. G. (2009). Satifación de los usuarios: Investigacón sobre la necesidades de información. México: Universidad Autonoma de México.

Chiavenato, I. (2002). Gestion del Talento Humano. Mc Graw Hill.

Chiavenato, I. (2007). Administración de los Recursos
Humanos. Mexico: McGraw-Hill.

Erskine Canelo, G. (2016). Fundamentos de la administración 2016 (1er parte). Obtenido de : http://www.slideshare.net/AlejandroGomez187/ fundamentos-de-la-administracin-2016- 1erparte-59657321.

Fandiño, M. (2014). Comites de Agua Potable Orgnizacion y Experiencias en Zona Secas, 1, 7- 13. Geilfus, F. (1997). 80 Herramientas para el Desarrollo Participativo. San Salvador, El Salvador:

Hernández Sampieri, R., Fernandez, C. C., \& Baptista, P. (2014). Metodologia de la Investigacíón. México: Mc Graw Hill Interamaerica Editores S.A de C.V.

Poder Legislativo. (14 de Junio de 2010). Ley 722 - Ley Especial de Comites de Agua Potable y Saneamiento. Gaceta Diario Oficial No. 111, pág. 5.

Posorky, R. (1996). "Photovoltaic water pumps, an attractive tool for rural drinking water supply". Solar Energy, 58, 155-163.

Zavala, J. M. (Agosto de 2016). Tesis sobre Organizacion y Gestion Comunitaria del Agua para Uso Domestico. Palacaguina. 